\title{
Combined Hybrid DFE and CCK Remodulator for Medium-Range Single-Carrier Underwater Acoustic Communications
}

\author{
Xialin Jiang, Wei Su, and En Cheng \\ Key Laboratory of Underwater Acoustic Communication and Marine Information Technology, Ministry of Education, \\ Xiamen University, Xiamen, Fujian 361005, China \\ Correspondence should be addressed to Wei Su; suweixiamen@xmu.edu.cn
}

Received 17 October 2016; Accepted 18 January 2017; Published 15 February 2017

Academic Editor: Michael McGuire

Copyright (C) 2017 Xialin Jiang et al. This is an open access article distributed under the Creative Commons Attribution License, which permits unrestricted use, distribution, and reproduction in any medium, provided the original work is properly cited.

\begin{abstract}
Advanced modulation and channel equalization techniques are essential for improving the performance of medium-range singlecarrier underwater acoustic communications. In this paper, an enhanced detection scheme, hybrid time-frequency domain decision feedback equalizer (DFE) combined with complementary code keying (CCK) remodulator, is presented. CCK modulation technique provides strong tolerance to intersymbol interference caused by multipath propagation in underwater acoustic channels. The conventional hybrid DFE, using a frequency domain feedforward filter and a time domain feedback filter, provides good performance along with low computational complexity. The error propagation in the feedback filter, caused by feedbacking wrong decisions prior to CCK demodulation, may lead to great performance degradation. In our proposed scheme, with the help of CCK coding gain, more accurate remodulated CCK chips can be used as feedback. The proposed detection scheme is tested by the practical ocean experiments. The experimental results show that the proposed detection scheme ensures robust communications over 10-kilometre underwater acoustic channels with the data rate at $5 \mathrm{Kbits} / \mathrm{s}$ in $3 \mathrm{kHz}$ of channel bandwidth.
\end{abstract}

\section{Introduction}

Underwater wireless communications are crucial for many civilian and military applications, such as offshore exploration, commercial fishing, pollution control, environmental monitoring, disaster prevention, and military missions [1]. Typical physical carriers for underwater wireless communication signals are radio waves, optical waves, and acoustic waves. Due to the strong absorption and attenuation, radio waves propagate poorly in underwater. Underwater radio communications, requiring the use of extremely low frequencies $(30-300 \mathrm{~Hz})$, large antenna systems, and high transmitter powers, are generally used over the very short range (up to ten meters). Underwater optical communications can provide extra high data rates (on the order of Mbps to Gbps), but again limited in very short distance (up to several tens of meters) because of rapid scattering. Instead, acoustic waves are the most attractive solution to longer (up to hundreds of kilometres) range underwater transmission since they suffer from relatively low absorption [2].
Underwater acoustic (UWA) communications, however, are severely affected by multipath propagation, frequencyselective fading, limited available bandwidth, Doppler spread, and ambient ocean noise. The underwater acoustic channel is generally recognized as one of the most challenging channels. The available bandwidth for underwater acoustic communication is severely limited due to attenuation that increases with signal frequency. For example, only about $1 \mathrm{kHz}$ bandwidth is available for $100 \mathrm{~km}$ range or $40 \mathrm{kHz}$ for $10 \mathrm{~km}$ range. The long multipath delay spread may extend over tens or even hundreds of symbols, causing severe intersymbol interference (ISI). The slow propagation speed of sound in water and the motion of the transmitter or receiver result in rapid time variation. Together, these impediments result in high-speed reliable communications over the underwater acoustic channel being very challenging [3]. Therefore, the advanced signal processing techniques are required to deal with high bit error rates, fading, and ISI for underwater acoustic communications [4]. 
In order to improve the performance of data detection and obtain the high-speed transmission over the bandlimited underwater acoustic channels, orthogonal frequencydivision multiplex (OFDM) has been applied as effective antimultipath technique for UWA communications [5, 6]. With the very special structure, the OFDM system can efficiently combat ISI. Additionally, another key feature, applying a low complexity frequency domain equalization (FDE) method via Discrete Fourier Transform (DFT) operation, has made OFDM system popular in communication systems. However, high peak-to-average-power ratio (PAPR) and sensitivity to carrier frequency offset (CFO), which causes intercarrier interference (ICI), are major challenges to OFDM system. An alternative to the OFDM approach, more traditional single-carrier (SC) system with advanced modulation and detection techniques, has been applied for UWA communication, which has comparable performance and low complexity [7]. SC system uses a single carrier, instead of multicarriers in OFDM system. Therefore, the peek-to-average power is much lower for SC system compared with OFDM system.

Over the past three decades, research in UWA communication has been active on modulation and detection techniques. In this paper, we investigate the complementary code keying (CCK) modulation technique for UWA communications [8], which has good autocorrelation and Euclidean distance properties to make strong tolerance to multipath distortion. Since UAW communications are characterized by the long delay spread caused by multipath propagation, the channel equalization is essential for successful detection of CCK modulation scheme [9-11]. Various time domain equalizers (TDE) have been extensively studied in the past $[12,13]$. It is well known that a maximum likelihood sequence equalizer (MLSE) gives optimum performance. The MLSE tests all possible data sequences and selects the data with the maximum probability as the output, but it is not practical for long channel delay spread, since the computational complexity of the MLSE increases exponentially with channel delay spread and signal constellation size. As a result, many suboptimal equalization methods have been proposed over the past few decades. The linear equalizer, such as minimum mean-squared error (MMSE) equalizer which minimizes the error between the received data and the transmitted data, is much simpler to implement but may suffer from significant performance loss compared to a nonlinear equalizer. The nonlinear decision feedback equalizer (DFE) based on zeroforcing or MMSE criterion often provides a good tradeoff between performance and complexity as the suboptimal solution when the signal constellation size is large and the channel delay spread is long. However, for transmissions over long delay spread underwater acoustic channel, TDE usually requires high computational complexity. Compared to TDE, frequency domain equalization (FDE) can effectively reduce the computational complexity by converting the received data from time domain to frequency domain [14-19]. Similar to OFDM, the FDE in SC system is conducted block-byblock via DFT and inverse DFT (IDFT) operation. Hence, SC system using FDE can achieve similar complexity and performance as OFDM system.
In receiver end, conventional detection schemes for CCK transmission separate channel equalization and CCK demodulation. However, the symbols from the output of the DFE before the CCK demodulation may be highly unreliable. If these wrong decisions are taken into the feedback loop, the performance will greatly degrade. In this paper, we propose an enhanced detection scheme, called hybrid time-frequency domain decision feedback equalizer (DFE) combined with complementary code keying (CCK) remodulator. In the proposed scheme, the frequency domain feedforward filter is used to reduce the computational complexity. The CCK coding gain is also exploited to feedback more reliable decoded decisions for error propagation cancellation.

The rest of the paper is organized as follows. In Section 2, we introduce CCK modulation and demodulation. A system model for CCK transmission over underwater acoustic channels is given in Section 3. In Section 4, we describe channel equalization techniques for CCK transmission. We present experimental results and discussions in Section 5. Finally, we draw conclusions in Section 6.

Throughout this paper, regular lower- or uppercase letters denote scalars, bold lower case letters denote column vectors, bold upper case letters denote matrices, the superscript $(\cdot)^{T}$ denotes matrix transpose, and $(\cdot)^{H}$ denotes matrix Hermitian.

\section{Complementary Code Keying}

Complementary codes were originally conceived by Golay in 1961 [20]. In order to achieve higher multiple data rate, the IEEE 802.11b standards committee has adopted CCK as the modulation scheme to deliver data rates of $5.5 \mathrm{Mbps}$ and $11 \mathrm{Mbps}$ in the $2.4 \mathrm{GHz}$ ISM band [21]. In this paper, we only consider the $11 \mathrm{Mbps}$ mode of CCK modulation because it is most relevant for high data rate applications.

2.1. CCK Modulation. CCK is an $M$-ary orthogonal keying modulation method, where one of the $M$ different code words which are nearly orthogonal to each other is chosen for use in transmission. For the eight complex chips CCK modulation, the CCK code words are derived according to the following formula [21]:

$$
\begin{aligned}
\mathbf{c}= & {\left[c_{1}, c_{2}, c_{3}, c_{4}, c_{5}, c_{6}, c_{7}, c_{8}\right] } \\
& =e^{j \varphi_{1}}\left[e^{j\left(\varphi_{2}+\varphi_{3}+\varphi_{4}\right)}, e^{j\left(\varphi_{3}+\varphi_{4}\right)}, e^{j\left(\varphi_{2}+\varphi_{4}\right)},\right. \\
& \left.-e^{j \varphi_{4}}, e^{j\left(\varphi_{2}+\varphi_{3}\right)}, e^{j \varphi_{3}},-e^{j \varphi_{2}}, 1\right],
\end{aligned}
$$

where $\mathbf{c}$ is the code word. As shown in (1), the CCK code word is determined by the four phase parameters $\varphi_{1}, \varphi_{2}, \varphi_{3}$, and $\varphi_{4}$, where $\varphi_{1}$ is contained in all eight chips, $\varphi_{2}$ is contained in all the odd chips, $\varphi_{3}$ is contained in all odd pairs of chips, and $\varphi_{4}$ is contained in all odd quads of chips. To make eight-chip CCK modulation, the input information bits are partitioned into 8-bit block as $\left(d_{1}, d_{2}, \ldots, d_{8}\right)$. The eight bits encode the four phase parameters according to the scheme shown in Table 1 [22]. 
TABLE 1: Phase parameters encoding scheme.

\begin{tabular}{lc}
\hline Dibit & Phase parameter \\
\hline$d_{2} d_{1}$ & $\varphi_{1}$ \\
$d_{4} d_{3}$ & $\varphi_{2}$ \\
$d_{6} d_{5}$ & $\varphi_{3}$ \\
$d_{8} d_{7}$ & $\varphi_{4}$ \\
\hline
\end{tabular}

TABLE 2: DQPSK encoding.

\begin{tabular}{lcc}
\hline$d_{2} d_{1}$ & $\varphi_{1}$ of even code word & $\varphi_{1}$ of odd code word \\
\hline 00 & 0 & $\pi$ \\
01 & $\pi / 2$ & $-\pi / 2$ \\
11 & $\pi$ & 0 \\
10 & $-\pi / 2$ & $\pi / 2$ \\
\hline
\end{tabular}

TABLE 3: QPSK encoding.

\begin{tabular}{lc}
\hline Dibit $\left(d_{i+1} d_{i}\right)$ & Phase \\
\hline 00 & 0 \\
01 & $\pi / 2$ \\
10 & $\pi$ \\
11 & $-\pi / 2$ \\
\hline
\end{tabular}

Once again, the 8-bit block data are divided into two bits and six bits. The first two bits $d_{0}$ and $d_{1}$ are used to modulate the phase $\varphi_{1}$ based on differential quadrature phase shift keying (DQPSK) modulation as specified in Table 2 [21]; that is, the phase $\varphi_{1}$ is relative to the phase $\varphi_{1}$ in the previous code word.

The other six dibits $\left(d_{4} d_{3}\right),\left(d_{6} d_{5}\right)$, and $\left(d_{8} d_{7}\right)$ are used to modulate the phase $\varphi_{2}, \varphi_{3}$, and $\varphi_{4}$ based on quadrature phase shift keying (QPSK) modulation, respectively, as shown in Table 3 [21].

For a code word length of eight complex chips CCK modulation, each chip is mapped to QPSK constellation. Each QPSK chip has four possible quadrature phases. Therefore, there are a total of $4^{8}=65536$ possible code words for CCK. However, CCK modulation takes eight information bits to define a code word of eight complex chips, so that each code word can carry eight bits data in transmission. That means CCK modulation choose sets of $2^{8}=256$ nearly orthogonal code words from 65536 possible code words pool. Hence, with the large set of good code words to pick, CCK modulation can obtain the significant coding distance and provide strong tolerance to multipath distortion.

\subsection{CCK Demodulation. At the receiver, CCK demodulation} is performed after channel equalization. CCK demodulation procedure is described in Figure 1.

To demodulate a CCK code word, in principle, we would correlate each received eight-chip CCK code word with all possible 256 CCK code words and then pick the largest correlation which corresponds to the code word transmitted. Referring to (1), we see that phase parameter $\varphi_{1}$ is contained in all eight chips of a code word, so it simply rotates the entire code word. That is, each chip of a code word would

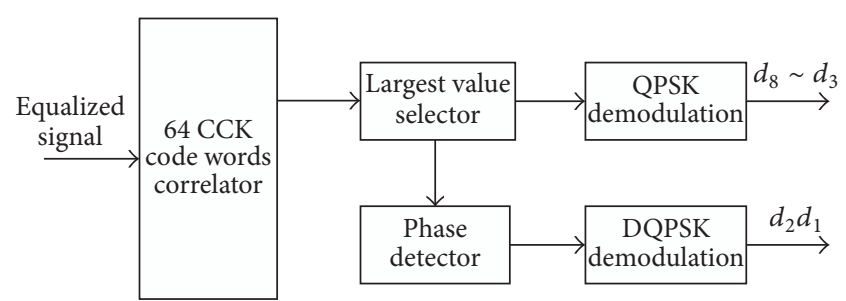

Figure 1: The CCK demodulation.

be a function of just $\varphi_{2}, \varphi_{3}$, and $\varphi_{4}$. Therefore, we would need only a bank of 64 correlators and a phase detection for a CCK code word demodulation. An incoming equalized eight chips code word is first correlated with 64 candidate base CCK code words. The correlation can be significantly simplified by using techniques like the Fast Walsh transform. The largest value among the 64 cross-correlation values would be selected to represent the demodulated code word; thereby the 6 bits of the code word transmitted are extracted after QPSK demodulation. The phase of this largest value would provide the other 2 bits of the 8-bit code word after DQPSK demodulation.

\section{System Model}

In this section, we describe the system model of CCK transmission over UWA channel. The block diagram of the CCK transmission system over UWA is shown in Figure 2.

At the transmitter, the stream of information data is encoded and modulated by the CCK modulator. The binary input data are mapped to QPSK symbols using formula (1). The blocking module produces data blocks. Then, a cyclic prefix (CP) is appended to each block, which is known at the receiver. A training sequence is followed by the data blocks, which is used for channel estimation. To reduce the channel bandwidth, a phase-shaping filter is applied to each of the transmitted data symbols. A synchronization signal is inserted in front of the packet, which is used to find the start and the end of transmitted signal. The packet structure of transmitted signal is shown in Figure 3. After the operation of digital-to-analog (D/A) conversion, signal amplification, the resulting sound waves converted by a transducer are transmitted over an underwater acoustic channel.

At the receiver, the signal at the output of the underwater acoustic channel performs transducer conversion (sound wave to the electrical signal), signal amplification, analogto-digital (A/D) conversion, and frequency downconversion. After time synchronization, the start of the transmitted signal is found; then the received noisy signal whose length equals the transmitted signal is extracted. The sample of each received data symbol is obtained by a phase-shaping filter at the receiver end. The received training sequence which is known at the receiver end is used for channel estimation. The received data symbols are divided into data blocks. Then the blocks are sent to the DFT module which converts the time domain data to the frequency domain. This is followed by frequency domain equalization. The equalized data blocks are 


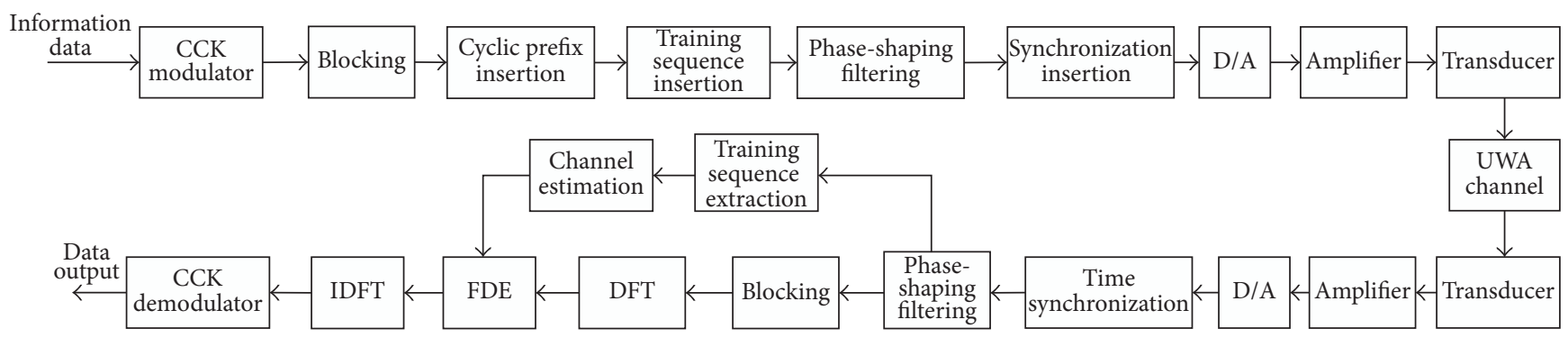

FIGURE 2: Block diagram of the CCK transmission system over UWA channel.

\begin{tabular}{|c|c|c|c|c|c|c|c|}
\hline Synchronization & Training & \multicolumn{4}{c|}{ Data payload } \\
\hline CP & Info block 1 & CP & Info block 2 & $\ldots$ & CP & Info block $i$ & $\cdots$ \\
\hline
\end{tabular}

FIGURE 3: The packet structure of the transmitted signal.

converted back to time domain by the IDFT module. Finally, the decisions of binary information data are made by the CCK demodulator.

\section{Equalization for CCK Transmission}

Underwater acoustic communications are severely affected by multipath propagation, which arises from sound reflections from the water surface, bottom and other objects, and sound refraction caused by sound speed variations with depth and temperature in the water. Multipath propagation causes severe long delay spread, as it generates intersymbol interference. Hence, the channel equalization is essential for successful detection of CCK transmission. For transmissions over long delay spread underwater acoustic channel, time domain equalization technique suffers from heavy computational complexity. Compared to time domain equalization, frequency domain equalization can effectively reduce the computational complexity by converting the received data from time domain to frequency domain via DFT operation.

4.1. Frequency Domain Linear Equalization. To implement the frequency domain equalization at the receiver end, the stream of transmitting data should be blockwise. Generally, a guard interval of a length equal to or greater than the duration of the channel impulse response is inserted at the beginning of each block. It not only acts as a guard interval that prevents interblock interference, but also plays a key role in the task of frequency domain equalization. Due to the insertion of a cyclic prefix, the linear convolution between transmitted data blocks and the channel impulse response is turned to a circular convolution, thus enabling blockby-block processing at the receiver end. Consequently, for the single carrier communication system, frequency domain equalization can be performed on blocks using the DFT operation, applying equalization to mitigate the ISI effects in the frequency domain, and converting the results back to the time domain via IDFT operation.

Let $\mathbf{x}=\left[x_{1}, x_{2}, \ldots, x_{N}\right]^{T}$ denote the $N$ symbols in one transmitted data block with the average energy being $\sigma_{x}^{2}$. The data block is transmitted over the underwater acoustic channel with the length of $M$ impulse response $\mathbf{h}=\left[h_{1}, h_{2}, \ldots, h_{M}\right]^{T}$. Although the underwater acoustic channel may actually be time-varying, if the variations are much slower than the data rate, the underwater acoustic channel can be assumed constant over the duration of a data block time scale. Moreover, we assume that downconversion, carrier synchronization, and timing recovery are operated flawlessly at the receiver end. The corresponding received data block $\mathbf{y}=\left[y_{1}, y_{2}, \ldots, y_{N}\right]^{T}$ can be written in a vector and matrix form as

$$
\mathbf{y}=\mathbf{H x}+\mathbf{v}
$$$$
\left[\begin{array}{c}
y_{1} \\
y_{2} \\
\vdots \\
\vdots \\
\vdots \\
\vdots \\
y_{N}
\end{array}\right]
$$ 


$$
=\left[\begin{array}{ccccccccc}
h_{1} & 0 & \cdots & 0 & h_{M} & h_{M-1} & h_{M-2} & \cdots & h_{2} \\
h_{2} & h_{1} & \ddots & 0 & 0 & h_{M} & h_{M-1} & \cdots & h_{3} \\
\vdots & \vdots & \ddots & \ddots & \ddots & \ddots & \ddots & \cdots & \vdots \\
h_{M} & h_{M-1} & h_{M-2} & \ddots & \ddots & h_{1} & 0 & \cdots & 0 \\
0 & 0 & \ddots & \ddots & \ddots & \ddots & \ddots & \ddots & 0 \\
\vdots & \vdots & \ddots & \ddots & \ddots & \ddots & \ddots & \ddots & \vdots \\
0 & 0 & \cdots & h_{M} & h_{M-1} & h_{M-2} & h_{M-3} & \cdots & h_{1}
\end{array}\right]\left[\begin{array}{c}
x_{1} \\
x_{2} \\
\vdots \\
\vdots \\
\vdots \\
\vdots \\
\vdots \\
\vdots \\
\vdots \\
\end{array}\right]
$$$$
+\left[\begin{array}{c}
v_{1} \\
v_{2} \\
\vdots \\
\vdots \\
\vdots \\
\vdots \\
v_{N}
\end{array}\right]
$$

where $\mathbf{H}$ is the time domain channel matrix. It is an $N$-by$N$ circular square matrix whose first column is given by the channel impulse response $\mathbf{h}$ with a number of zeros padding to the end to extend its length to the block size of $N$. It is assumed that the size of block $N$ is larger than the length of channel impulse response $M . \mathbf{v}=\left[v_{1}, v_{2}, \ldots, v_{N}\right]^{T}$ is the additive white Gaussian noise with the variance equal to $\sigma_{v}^{2}$.

According to the feature of circular matrices, every circular matrix can be diagonalized by the DFT matrix. The $N$-by- $N$ DFT matrix is defined as

$$
\begin{aligned}
\mathbf{F} & =\frac{1}{\sqrt{N}} \\
& \left(\begin{array}{ccccc}
1 & 1 & 1 & \cdots & 1 \\
1 & e^{-j(2 \pi / N)} & e^{-j(4 \pi / N)} & \cdots & e^{-j(2 \pi(N-1) / N)} \\
1 & e^{-j(4 \pi / N)} & e^{-j(8 \pi / N)} & \cdots & e^{-j(4 \pi(N-1) / N)} \\
\vdots & \vdots & \vdots & \ddots & \vdots \\
1 & e^{-j(2 \pi(N-1) / N)} & e^{-j(4 \pi(N-1) / N)} & \cdots & e^{-j\left(2 \pi(N-1)^{2} / N\right)}
\end{array}\right) .
\end{aligned}
$$

If we left-multiply a proper DFT matrix and rightmultiply the corresponding IDFT matrix with the circular matrix $\mathbf{H}$, then a diagonal matrix can be obtained. As such, we get

$$
\Lambda=\mathbf{F H F}^{H},
$$

where $\Lambda$ is an $N$-by- $N$ diagonal matrix whose eigenvalues are also given by taking the DFT of the first column of circular channel matrix $\mathbf{H}$, and $\mathbf{F}^{H}$ is the corresponding matrix performing the IDFT operation. It is easy to confirm that $\mathbf{F}^{H} \mathbf{F}=\mathbf{I}$, where $\mathbf{I}$ is the $N$-by-by- $N$ identity matrix.
We define the frequency domain vectors as

$$
\begin{aligned}
& \mathbf{Y}=\mathbf{F y}, \\
& \mathbf{X}=\mathbf{F} \mathbf{x}, \\
& \mathbf{V}=\mathbf{F v} .
\end{aligned}
$$

The received data block in the frequency domain can be obtained by multiplying DFT matrix $\mathbf{F}$ to both sides of (2). As such, we get

$$
\begin{aligned}
\mathbf{F y} & =\mathbf{F H F}^{H} \mathbf{F x}+\mathbf{F v}, \\
\mathbf{Y} & =\mathbf{\Lambda} \mathbf{X}+\mathbf{V} .
\end{aligned}
$$

From (2) and (6), we can see that the time domain circular channel matrix is turned to a frequency domain diagonal channel matrix, so the frequency domain equalization will only have to deal with a diagonal matrix. Since the inverse of a diagonal matrix is obtained by replacing each element in the diagonal with its reciprocal, the computational complexity for frequency domain equalization is largely reduced. Moreover, the DFT and IDFT operation can be replaced by the efficient algorithms fast Fourier transform (FFT) and inverse FFT (IFFT) algorithms to further reduce the complexity.

The entries of the diagonal channel matrix $\Lambda$ are $\lambda=$ $\left[\lambda_{1}, \lambda_{2}, \ldots, \lambda_{N}\right]$, which can be denoted by the channel attenuations in the frequency domain. The simplest frequency domain equalization can be achieved by simply pointwise division of the received data block by the corresponding channel attenuations. This method is called zero-forcing (ZF) frequency domain equalization since the ISI at the equalizer output is forced to zero, which can be expressed as

$$
\mathbf{X}=\Lambda^{-1} \mathbf{Y}
$$

From (7), we can see that the ZF frequency domain equalization ignores the presence of additive white Gaussian noise. But the noise is always present. ZF equalization may lead to great noise enhancements at the frequencies where the channel frequency response has spectral nulls. Although the ZF equalization removes the ISI, the great noise enhancements will still result in poor performance. In order to avoid noise enhancements, an alternative is the minimum mean-squared error (MMSE) equalizer which minimizes the error between the transmitted symbols and the output of the equalizer. It is based on the mean square error (MSE) criterion. The MMSE equalizer takes noises into account, which requires the second-order statistical information about the transmitted data block and the noise signal. We assume that the $N$ transmitted data block has uniform power. Here note that in (6) $E\left\{\mathbf{X X} \mathbf{X}^{H}\right\}=\sigma_{x}^{2} \mathbf{I}$ and $E\left\{\mathbf{V} \mathbf{V}^{H}\right\}=\sigma_{v}^{2} \mathbf{I}$. It provides a good trade-off between ISI removal and noise enhancement. Therefore, the MMSE equalizer achieves a better performance compared to ZF equalizers at low SNRs. The frequency domain of MMSE equalization can be expressed as

$$
\mathbf{X}=\left(\boldsymbol{\Lambda} \boldsymbol{\Lambda}^{*}+\frac{\sigma_{v}^{2}}{\sigma_{x}^{2}} \mathbf{I}\right)^{-1} \boldsymbol{\Lambda} \mathbf{Y}
$$




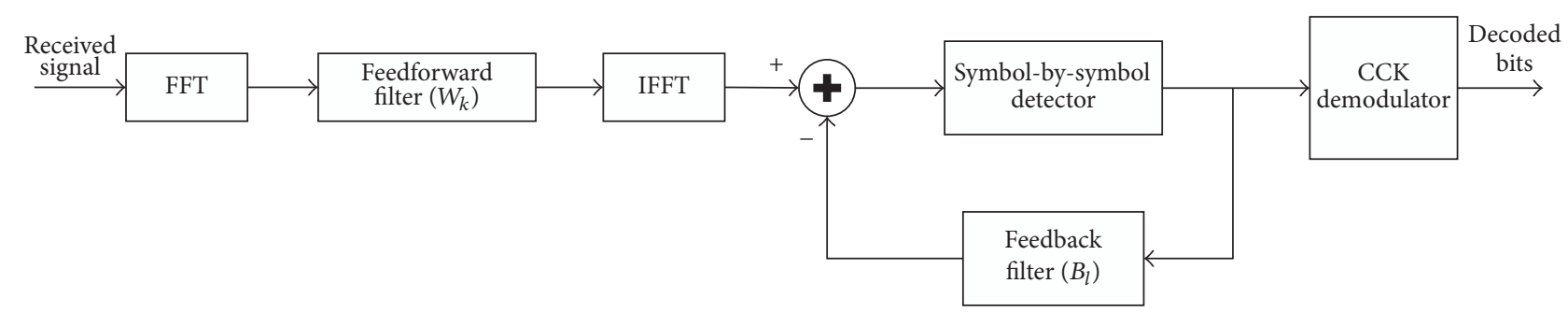

FIGURE 4: The block diagram of the hybrid time-frequency domain DFE followed by CCK demodulator.

4.2. Hybrid DFE Combined with CCK Remodulator. Frequency domain ZF and MMSE linear equalization are quite simple to implement, since the inversion of a diagonal matrix is easy to calculate. However, for underwater acoustic channels, the probability of spectral nulls is very high, and the SNRs at the receiver end are very low. Therefore, linear ZF and MMSE equalizers are not directly employed for underwater acoustic channels. A good choice for underwater acoustic channels with severe ISI is a nonlinear equalizer. To improve the performance of frequency domain linear equalization, we consider the hybrid time-frequency domain equalization with decision feedback for CCK transmission. The block diagram of the hybrid time-frequency domain DFE followed by CCK demodulator is shown in Figure 4.

The hybrid time-frequency domain DFE consists of two filters using a frequency domain filtering only for the feedforward filter and a time domain filtering for the feedback filter. A block of $N$ received ISI affected signals are transformed to the frequency domain by the FFT module. The FFT output is first weighted with the $N$-tap feedforward filter coefficients $\mathbf{W}=\left[W_{1}, W_{2}, \ldots, W_{N}\right]^{T}$. The resulting signals are then transformed back to the time domain by the IFFT module. The trailing ISI is removed by the $N_{b}$-tap $\mathbf{B}=\left[B_{1}, B_{2}, \ldots, B_{N_{b}}\right]^{T}$ time domain feedback filter using previously decided symbols. The output of feedback filter is subtracted from the output of IFFT to form the estimated symbol; then the symbol-by-symbol detector makes the decision for each of the estimated symbols. The coefficients of the frequency domain feedforward filter and the time domain feedback filter can be obtained by using MMSE criterion. In a similar way to (8), the coefficients of the feedforward filter [17] can be calculated as

$$
\begin{aligned}
& W_{k}=\frac{\sigma_{x}^{2} \lambda_{k}\left(1+\sum_{l=1}^{N_{b}} B_{l} e^{-j 2 \pi(k-1) l / N}\right)}{\sigma_{x}^{2}\left|\lambda_{k}\right|^{2}+\sigma_{v}^{2}}, \\
& k=1,2, \ldots, N
\end{aligned}
$$

The coefficients of the feedback filter can be obtained by solving the number of $N_{b}$ linear equations, which can be written in the vector and matrix form as

$$
\mathrm{AB}=\boldsymbol{\alpha},
$$

where the matrix $\mathbf{A}=\left[A_{i j}\right]$ and the column vector $\boldsymbol{\alpha}=\left[\alpha_{1}\right.$, $\left.\alpha_{2}, \ldots, \alpha_{N_{b}}\right]^{T}$ are defined as

$$
\begin{aligned}
A_{i j} & =\sum_{k=1}^{N} \frac{\sigma_{x}^{2} e^{-j 2 \pi(k-1)(j-i) / N}}{\sigma_{x}^{2}\left|\lambda_{k}\right|^{2}+\sigma_{v}^{2}}, \quad i \text { or } j=1,2, \ldots, N_{b}, \\
\alpha_{m} & =\sum_{k=1}^{N} \frac{\sigma_{x}^{2} e^{j 2 \pi(k-1) m / N}}{\sigma_{x}^{2}\left|\lambda_{k}\right|^{2}+\sigma_{v}^{2}}, \quad m=1,2, \ldots, N_{b} .
\end{aligned}
$$

As from (11), we can see that the matrix $\mathbf{A}$ is an $N_{b}$-by- $N_{b}$ Toeplitz matrix, and $\alpha_{m}$ is the IDFT of $\left\{\sigma_{x}^{2} /\left(\sigma_{x}^{2}\left|\lambda_{k}\right|^{2}+\sigma_{v}^{2}\right)\right\}$. Therefore, the efficient algorithm FFT can be used to solve problem (10) for the computational complexity reduction. Once the coefficients of the feedback filter are determined, submitting them to (9), the coefficients of feedforward filter can be given.

In the conventional detection method, the hybrid DFE and CCK demodulator are completely separated. The equalized data blocks are sent to the CCK demodulator for the decisions of binary data. In this method, the feedback symbols of hybrid DFE are the equalized symbols without the CCK demodulation. Due to the low SNR and the finite taps of hybrid DFE, these equalized symbols may be highly unreliable. If these equalized symbols are immediately used as the input of the feedback filter of hybrid DFE, this may lead to error propagation. If a symbol is incorrectly decided in hybrid DFE, the propagation of wrong decisions will last several symbols period. Hence, this will cause high performance degradation.

Since error propagation in hybrid DFE would cause severe performance degradation, the reliability of feedback symbols has to be concerned with. To further improve the performance, we propose an enhanced detection scheme, that is, the hybrid DFE combined with CCK remodulator. The block diagram of the hybrid DFE combined with CCK remodulator is shown in Figure 5.

Our basic idea is to combine the hybrid DFE and CCK demodulator together and feedback more reliable symbols to remove ISI. We understand that the interchip interference in one received CCK code word could be eliminated by the CCK demodulation. In order to reduce the error propagation phenomenon in the time domain feedback filter, we can take advantage of the CCK coding gain. Compared to the conventional separate detection scheme, in our proposed scheme, a CCK remodulator block is added at the receiver end, which remodulates the decoded data from the output of 


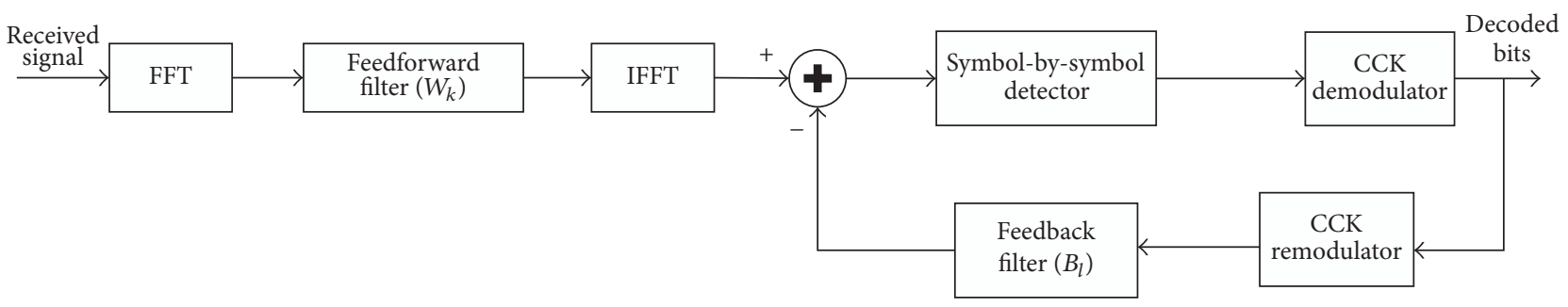

FIGURE 5: The block diagram of hybrid DFE combined with CCK remodulator.

the CCK demodulator and sends the output to the feedback filter of hybrid DFE. Since the input of feedback filter is from the CCK remodulator, the accuracy of feedbacked symbols is improved in contrast to feedbacking the equalized symbols prior to CCK demodulation. With more accurate feedbacked symbols, the error propagation in the feedback filter would be reduced. Hence, the performance of detection for CCK transmission will be greatly enhanced.

\subsection{Sparse Channel Estimation and Noise Variance Estima-} tion. The estimations of channel impulse response $\mathbf{h}$ and noise variance $\sigma_{v}^{2}$ are needed to the hybrid DFE design. Accurate channel impulse response estimation and noise variance estimation play an important role in the performance of the overall system. Conventional channel estimation algorithms require transmission of a training data sequence to estimate the channel characteristics. In this paper, we use two backto-back Gold sequences as training signal which is known to the receiver to perform channel characteristics estimation. The Gold sequence has special properties of autocorrelation and cross-correlation. In order to improve the accuracy of channel estimation, the first Gold sequence acts the role of the cyclic prefix, which absorbs intersymbol interference. The second Gold sequence is used for channel impulse response estimation and noise variance estimation.

Underwater acoustic channels are generally sparse, because most of the channel energy is located at a few spread delays. We implement the channel estimation using the matching pursuit (MP) algorithm [23, 24], which is very suitable for sparse underwater acoustic channel estimation. As mentioned above, the channel noise is modeled as additive Gaussian white with variance $\sigma_{v}^{2}$. We use the following approach to make the estimation of the channel noise variance. With the knowledge of transmitted training sequence $N_{p}$-by- $N_{p}$ Toeplitz matrix $\mathbf{S}$, the corresponding received training sequence $\mathbf{y}_{p}$, and the estimation of channel impulse response $\widehat{\mathbf{h}}$, noise variance estimation can be expressed as

$$
\widehat{\sigma}_{v}^{2}=\frac{1}{N_{p}}\left\|\mathbf{y}_{p}-\mathbf{S} \widehat{\mathbf{h}}\right\|^{2} .
$$

\section{Experiment Results and Discussions}

In this section, we evaluate the performance of the proposed hybrid DFE with combined CCK remodulator over the medium range shallow underwater acoustic channels. The ocean experimental data was collected off the Coast of
Xiamen, near the Taiwan Strait, Fujian, China. The sketch map of ocean test equipment arrangements is depicted in Figure 6.

In the ocean tests, we set up transmitter and receiver, respectively, on two ships, named as Marine I and Marine II. We used GPS devices on each ship to acquire the accurate location of two ships. The ocean conditions, such as water temperature and water depth, were also recorded when the tests were done. We set the direct distance between Marine I and Marine II about ten kilometres far. There was one transmitter set in the Marine II. The transducer as the transmitter was deployed nine meters beneath the water surface. The depth of water was about sixteen meters around the transmitter area. In Marine I, we arranged five transducers to receive the transmitted acoustic signals in the water. These five receivers were divided into two groups. Three transducers as receivers are grouped at the head of Marine I. They are equally spaced three meters with each other in a vertical array. The deepest one was set nine meters under water. They are labelled as R1, R2, and R3 from up to down, respectively, in Figure 6. The other two transducers as receivers are grouped at the tail of Marine I. They were also vertically set at 4.5 meters under water and 10.5 meters under water, respectively. They were labelled as R4 and R5 in Figure 6. The depth of water was about 20 meters around Marine I area. The data was collected by NI DAQ (NI USB-6259) converter which was connected to a computer. During the experiments, the received signals were stored in computer for later offline processing. Because of the uncontrolled conditions in the ocean, the good time for experiments was extremely limited.

The parameters in the experiments are set as follows. The carrier frequency is $6 \mathrm{kHz}$. The duration of one symbol is $T_{s}=0.4 \mathrm{~ms}$. The roll-off factor of pulse shaping filter is set to 0.2 . Hence, the occupied channel bandwidth is $3 \mathrm{kHz}$, which starts from $4.5 \mathrm{kHz}$ to $7.5 \mathrm{kHz}$. The sample rate is set to $60 \mathrm{kHz}$. Information bits are mapped to QPSK symbols according to CCK modulation formula. The data block size is set to $N=1024$. The length of CP is set to 256 . The training signal is made of two 512-bit Gold sequence (appending a zero in the end). A linear frequency modulation (LFM) signal sweeps over the whole transmit bandwidth used for time synchronization.

In Figure 7, the magnitude of received passband signal from transducer R1 is plotted. The time domain burst interferences in the received signal are observed from the figure, obviously. The power spectrum density (PSD) of the received signal from transducer R1 is depicted in Figure 8. 


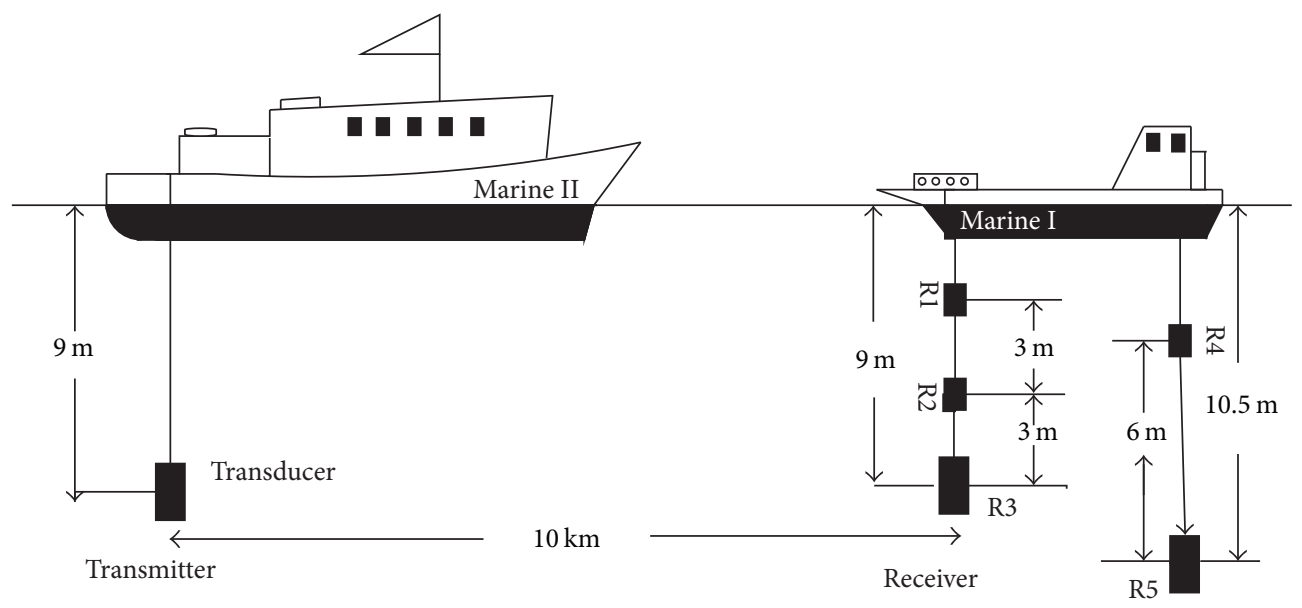

FIGURE 6: The deployment of equipment.

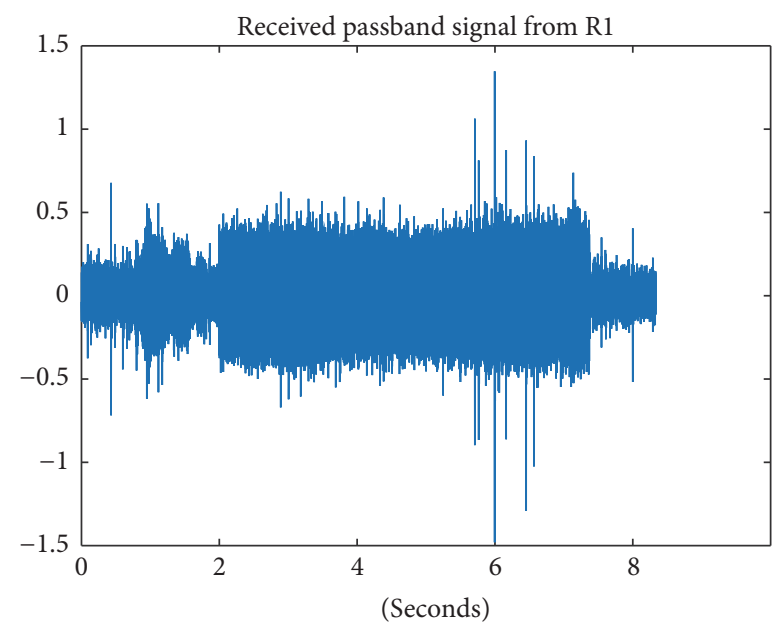

FIGURE 7: The received passband signal from transducer R1.

It is clear that the PSD of received signal spans over the channel bandwidth from $4.5 \mathrm{kHz}$ to $7.5 \mathrm{kHz}$ in the frequency domain, and the frequency components center at the carrier frequency $6 \mathrm{kHz}$. The dynamic range of PSD is more than $30 \mathrm{~dB}$ over the channel bandwidth. The transducers we used in the experiment have flat frequency response $( \pm$ $2 \mathrm{~dB}$ ) from $200 \mathrm{~Hz}$ to $10 \mathrm{kHz}$. It indicates that the frequency dependent attenuation is highly severe over the underwater acoustic channels. The time domain burst interferences and frequency domain uneven attenuations make the detection for CCK transmission over underwater acoustic channels very challenging.

As mentioned above, the received LFM signal can be used for synchronization. To find the synchronization point, the received signals are correlated with the local LFM signal. The peak of correlation is corresponding to the start of transmitted signal. In Figure 9, an example of the normalized LFM correlation is demonstrated for received signal from transducer R1 and R5. From the figure, the time span of the

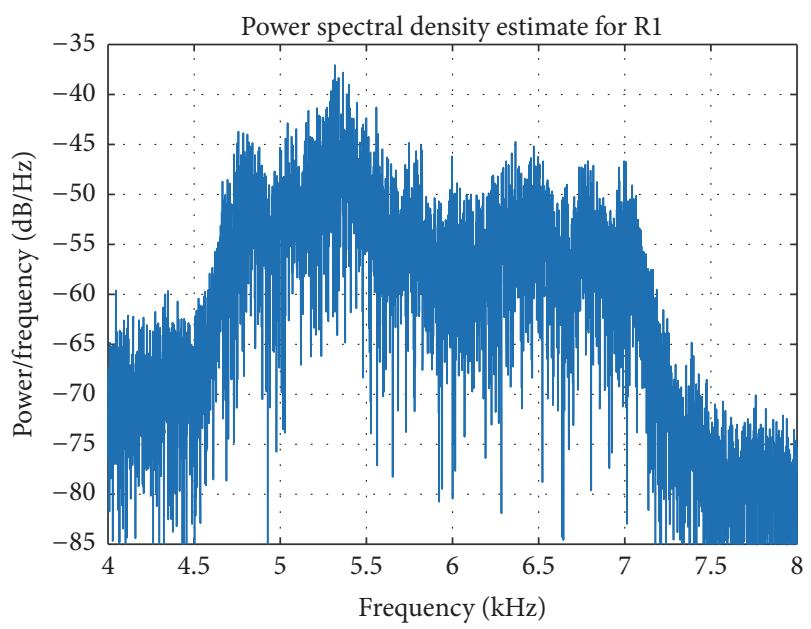

Figure 8: The power spectrum magnitude of received signals from transducer R1.

significant correlation is about $20 \mathrm{~ms}$. It indicates that the length of channel impulse response is about 50 in terms of the symbol period $T_{s}=0.4 \mathrm{~ms}$.

An example of the channel impulse response (CIR) for UWA channels is shown in Figure 10. Clearly, the channel impulse response has sparse multipath gain, which results in severe ISI at the receiver. This characteristic of the UWA channels makes the channel detection at the receiver very challenging.

Once the channel estimation and noise variance estimation are obtained, the equalization can be performed. We compare the performance of our proposed hybrid DFE combined with CCK remodulator with other three detection schemes: frequency domain (FD) MMSE equalizer followed by CCK demodulator and hybrid DFE with perfect feedback. We report the signal-to-noise ratio (SNR) at the output of the equalizer and bit error rate (BER) for four detection schemes in Tables 4 and 5, respectively. Since the mean energy of the 


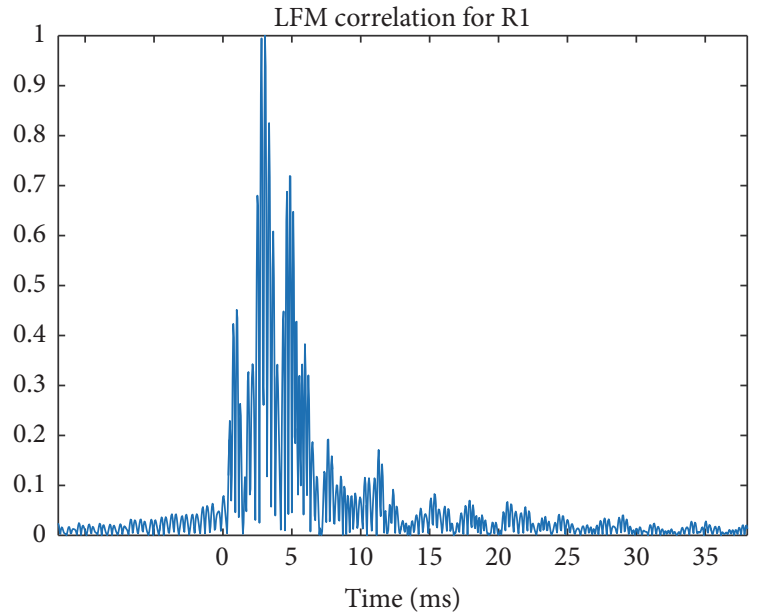

(a)

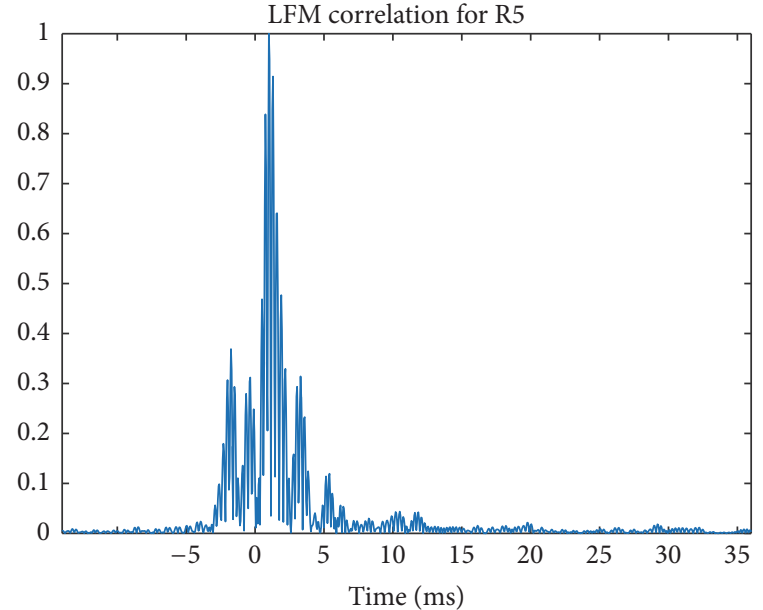

(b)

FIGURE 9: An example of the LFM correlation.

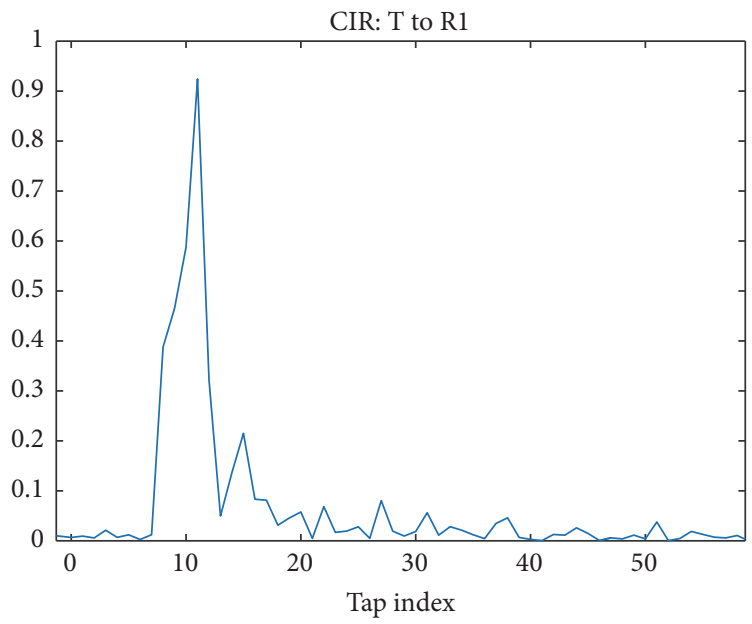

(a)

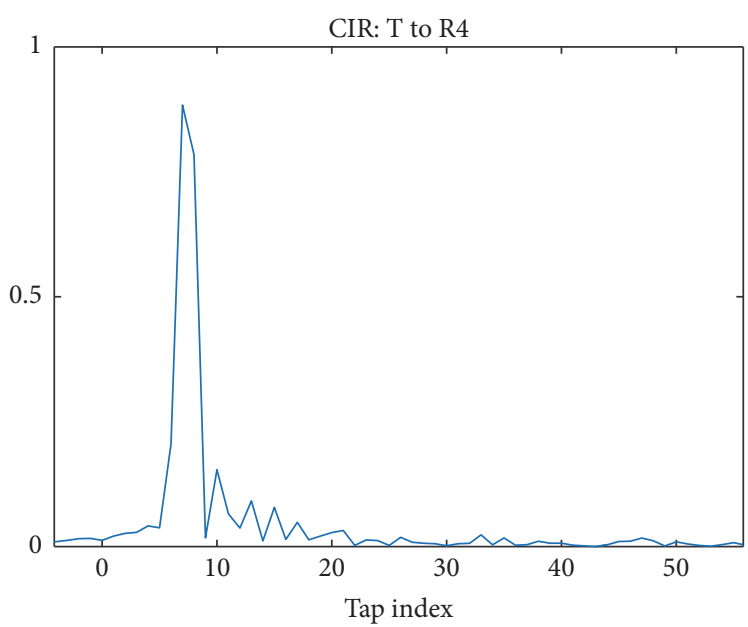

(c)

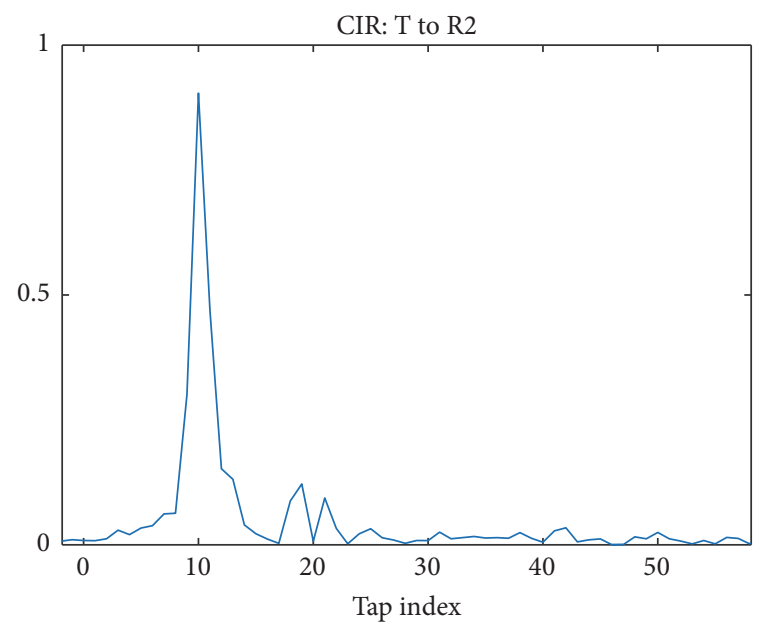

(b)

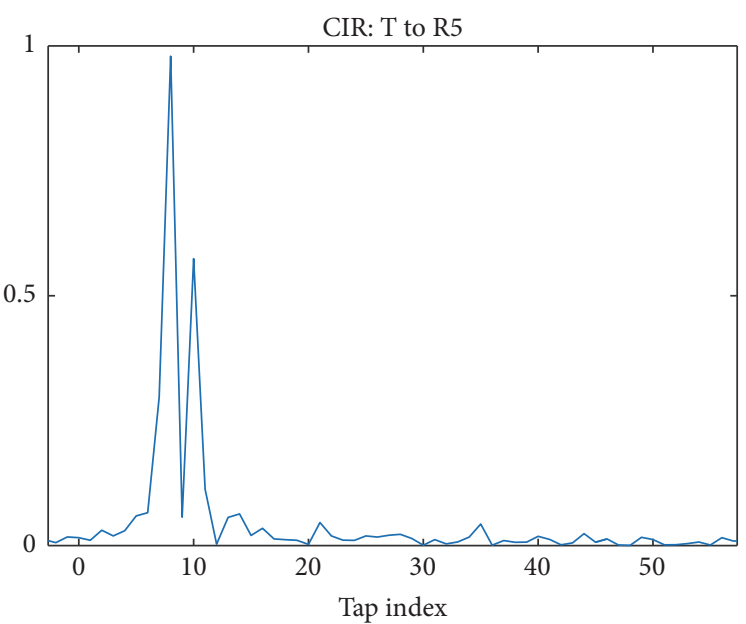

(d)

FIGURE 10: Example of estimated channel impulse response in the experiment (“T” denotes transmitter transducer). 
TABLE 4: The SNR at the output of equalizer for different detection schemes.

\begin{tabular}{lcccc}
\hline Transducer\# & $\begin{array}{c}\text { FD-MMSE followed } \\
\text { by CCK demodulator }\end{array}$ & $\begin{array}{c}\text { Hybrid DFE followed } \\
\text { by CCK demodulator }\end{array}$ & $\begin{array}{c}\text { Hybrid DFE combined } \\
\text { with CCK remodulator }\end{array}$ & $\begin{array}{c}\text { Hybrid DFE with } \\
\text { perfect feedback }\end{array}$ \\
\hline R1 & $1.12 \mathrm{~dB}$ & $1.61 \mathrm{~dB}$ & $1.96 \mathrm{~dB}$ & $2.01 \mathrm{~dB}$ \\
R2 & $1.43 \mathrm{~dB}$ & $4.03 \mathrm{~dB}$ & $5.30 \mathrm{~dB}$ & $5.30 \mathrm{~dB}$ \\
R3 & $1.38 \mathrm{~dB}$ & $3.87 \mathrm{~dB}$ & $4.71 \mathrm{~dB}$ & $4.75 \mathrm{~dB}$ \\
R4 & $1.28 \mathrm{~dB}$ & $2.33 \mathrm{~dB}$ & $2.63 \mathrm{~dB}$ & $2.68 \mathrm{~dB}$ \\
R5 & $1.86 \mathrm{~dB}$ & $6.68 \mathrm{~dB}$ & $7.84 \mathrm{~dB}$ & $7.84 \mathrm{~dB}$ \\
\hline
\end{tabular}

TABLE 5: BER results of different detection schemes.

\begin{tabular}{lcccc}
\hline Transducer\# & $\begin{array}{c}\text { FD-MMSE followed } \\
\text { by CCK demodulator }\end{array}$ & $\begin{array}{c}\text { Hybrid DFE followed } \\
\text { by CCK demodulator }\end{array}$ & $\begin{array}{c}\text { Hybrid DFE combined } \\
\text { with CCK remodulator }\end{array}$ & $\begin{array}{c}\text { Hybrid DFE with } \\
\text { perfect feedback }\end{array}$ \\
\hline R1 & $4.58 \times 10^{-1}$ & $3.87 \times 10^{-2}$ & $2.56 \times 10^{-2}$ & $2.42 \times 10^{-2}$ \\
R2 & $5.04 \times 10^{-2}$ & $9.76 \times 10^{-4}$ & $3.25 \times 10^{-4}$ & $<10^{-4}$ \\
R3 & $4.76 \times 10^{-2}$ & $5.69 \times 10^{-4}$ & $1.22 \times 10^{-4}$ & $<10^{-4}$ \\
R4 & $3.89 \times 10^{-1}$ & $1.53 \times 10^{-2}$ & $9.92 \times 10^{-3}$ & $2.44 \times 10^{-4}$ \\
R5 & $3.20 \times 10^{-2}$ & $<10^{-4}$ & $<10^{-4}$ & $<10^{-4}$ \\
\hline
\end{tabular}

transmitted symbols is normalized to 1 , the SNR at the output of the equalizer is defined as

$$
\mathrm{SNR}_{\text {out }} \triangleq \frac{E\left(\left|x_{n}\right|^{2}\right)}{E\left(\left|x_{n}-\widehat{x}_{n}\right|^{2}\right)}=\frac{1}{E\left(\left|x_{n}-\widehat{x}_{n}\right|^{2}\right)}
$$

where the output of the equalizer $\widehat{x}_{n}$ is the estimate of the transmitted symbol $x_{n}$ and $E\left(\left|x_{n}-\widehat{x}_{n}\right|^{2}\right)$ is the mean square error (MSE).

For the results shown in Tables 4 and 5, the proposed hybrid DFE combined with CCK remodulator achieves better performance compared to hybrid DFE followed by CCK demodulator scheme. This verifies that the performance of hybrid DFE in the proposed scheme is significantly enhanced. The accuracy of the feedbacked symbols is significantly improved through the CCK remodulator, which takes advantage of the CCK coding gain. The linear FD-MMSE equalizer cannot effectively mitigate channel distortions for these underwater acoustic channels, R1 and R4. Since they are both close to the water surface, they suffer from more interferences than the deeper deployed transducers. The proposed hybrid DFE combined with CCK remodulator scheme can get about 1.0 dB SNR improvement compared to the conventional hybrid DFE followed by CCK demodulator scheme. Also, for the data collected from transducers R2, R3, and $\mathrm{R} 5$, the proposed scheme can achieve the order of $10^{-4}$ BER transmission. This means that reliable communications over 10-kilometre underwater acoustic channels are obtained with the data rate $5 \mathrm{Kbits} / \mathrm{s}$ in $3 \mathrm{kHz}$ of channel bandwidth.

\section{Conclusion}

In this paper, three receiver detection schemes for CCK transmission have been tested with the collected data in real-world underwater acoustic communications experiments. Frequency domain equalization can largely reduce the computation complexity to deal with ISI caused by multipath propagation effects. The conventional hybrid DFE can achieve better performance than linear frequency domain MMSE for severely distorted underwater acoustic channels. However, the error propagation may occur in the feedback filter of hybrid DFE, because the unreliable symbols are feedbacked prior to CCK demodulation. In our proposed hybrid DFE combined with CCK remodulator scheme, with the help of CCK coding gain, more accurate remodulated CCK chips can be used as feedback. The practical ocean experimental results have demonstrated that the proposed hybrid DFE combined with CCK remodulator scheme achieves better BER performance than the conventional hybrid DFE followed by CCK demodulator scheme. Robust communications over 10-kilometre underwater acoustic channels have been achieved with the data rate at $5 \mathrm{Kbits} / \mathrm{s}$ in $3 \mathrm{kHz}$ of channel bandwidth. This demonstrates that our proposed detection scheme is an attractive approach in terms of computational complexity and performance for underwater acoustic communications.

\section{Competing Interests}

The authors declare that there is no conflict of interests regarding the publication of this paper.

\section{Acknowledgments}

The authors are thankful for the support from the National Natural Science Foundation of China (Grants nos. 61301097, 61671398 , and 61471308). Many thanks are due to the teachers and students who participated in the ocean experiments. The authors would like to thank Professor Ryan Kastner at University of California, San Diego, for his guidance and discussions. Xialin Jiang would like to acknowledge the fellowship of Postgraduate-Overseas Study Program for Building High-Level Universities from the China Scholarship Council. 


\section{References}

[1] D. Pompili and I. Akyildiz, "Overview of networking protocols for underwater wireless communications," IEEE Communications Magazine, vol. 47, no. 1, pp. 97-102, 2009.

[2] M. Stojanovic, "Acoustic (underwater) communications," in Encyclopedia of Telecommunications, John Wiley \& Sons, 2003.

[3] M. Chitre, S. Shahabudeen, and M. Stojanovic, "Underwater acoustic communications and networking: recent advances and future challenges," Marine Technology Society Journal, vol. 42, no. 1, pp. 103-116, 2008.

[4] A. C. Singer, J. K. Nelson, and S. S. Kozat, "Signal processing for underwater acoustic communications," IEEE Communications Magazine, vol. 47, no. 1, pp. 90-96, 2009.

[5] L. Wan, H. Zhou, X. Xu et al., "Adaptive modulation and coding for underwater acoustic OFDM," IEEE Journal of Oceanic Engineering, vol. 40, no. 2, pp. 327-336, 2015.

[6] A. Radosevic, R. Ahmed, T. M. Duman, J. G. Proakis, and M. Stojanovic, "Adaptive OFDM modulation for underwater acoustic communications: design considerations and experimental results," IEEE Journal of Oceanic Engineering, vol. 39, no. 2, pp. 357-370, 2014.

[7] Y. R. Zheng, J. Wu, and C. Xiao, "Turbo equalization for singlecarrier underwater acoustic communications," IEEE Communications Magazine, vol. 53, no. 11, pp. 79-87, 2015.

[8] C. He, J. Huang, and Z. Ding, "A variable-rate spread-spectrum system for underwater acoustic communications," IEEE Journal of Oceanic Engineering, vol. 34, no. 4, pp. 624-633, 2009.

[9] B.-H. Kim, "Bidirectional iterative ISI canceller for high-rate DSSS/CCK communications," IEEE Journal on Selected Areas in Communications, vol. 24, no. 1, pp. 171-180, 2006.

[10] Y. Lee and H. Park, "A RAKE receiver with an ICI/ISI equalizer for a CCK modem," IEEE Transactions on Vehicular Technology, vol. 58, no. 1, pp. 198-206, 2009.

[11] C. Jonietz, W. H. Gerstacker, and R. Schober, "Sphere constrained detection of complementary code keying signals transmitted over frequency selective channels," IEEE Transactions on Wireless Communications, vol. 8, no. 9, pp. 4656-4667, 2009.

[12] J. G. Proakis, Digital Communications, McGraw-Hill, New York, NY, USA, 1995.

[13] S. Haykin, Communication Systems, John Wiley \& Sons, 2008.

[14] F. Pancaldi, G. M. Vitetta, R. Kalbasi, N. Al-Dhahir, M. Uysal, and H. Mheidat, "Single-carrier frequency domain equalization," IEEE Signal Processing Magazine, vol. 25, no. 5, pp. 37-56, 2008.

[15] D. Falconer, S. L. Ariyavisitakul, A. Benyamin-Seeyar, and B. Eidson, "Frequency domain equalization for single-carrier broadband wireless systems," IEEE Communications Magazine, vol. 40, no. 4, pp. 58-66, 2002.

[16] N. Benvenuto, R. Dinis, D. Falconer, and S. Tomasin, "Single carrier modulation with nonlinear frequency domain equalization: an idea whose time has come-again," Proceedings of the IEEE, vol. 98, no. 1, pp. 69-96, 2010.

[17] N. Benvenuto and S. Tomasin, "On the comparison between OFDM and single carrier modulation with a DFE using a frequency-domain feedforward filter," IEEE Transactions on Communications, vol. 50, no. 6, pp. 947-955, 2002.

[18] Y. Zhu and K. B. Letaief, "Single carrier frequency domain equalization with time domain noise prediction for wideband wireless communications," IEEE Transactions on Wireless Communications, vol. 5, no. 12, pp. 3548-3557, 2006.
[19] L.-N. Tran, E.-K. Hong, and H. Liu, "Two-stage hybrid decision feedback equalization for DS-CDMA systems," IEEE Transactions on Wireless Communications, vol. 7, no. 5, pp. 1490-1494, 2008.

[20] M. Golay, “Complementary series", IRE Transactions on Information Theory, vol. 7, no. 2, pp. 82-87, 1961.

[21] Institute of Electrical and Electronics Engineers, Standard 802.11b-1999, Part 11: Wireless LAN Medium Access Control (MAC) and Physical Layer (PHY) Specifications: Higher-Speed Physical layer Extension in the 2.4 GHz Band, IEEE, 2000.

[22] B. Pearson, "Complementary code keying made simple," Application Notes AN9850.1, Intersil, Milpitas, Calif, USA, 2000.

[23] S. F. Cotter and B. D. Rao, "Sparse channel estimation via matching pursuit with application to equalization," IEEE Transactions on Communications, vol. 50, no. 3, pp. 374-377, 2002.

[24] A. Song, M. Badiey, V. K. McDonald, and T. C. Yang, "Time reversal receivers for high data rate acoustic multiple-inputmultiple-output communication," IEEE Journal of Oceanic Engineering, vol. 36, no. 4, pp. 525-538, 2011. 


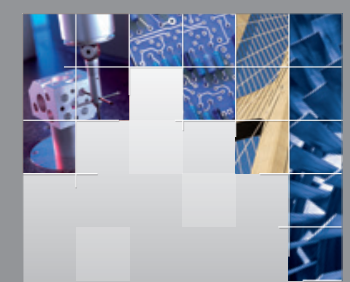

\section{Enfincering}
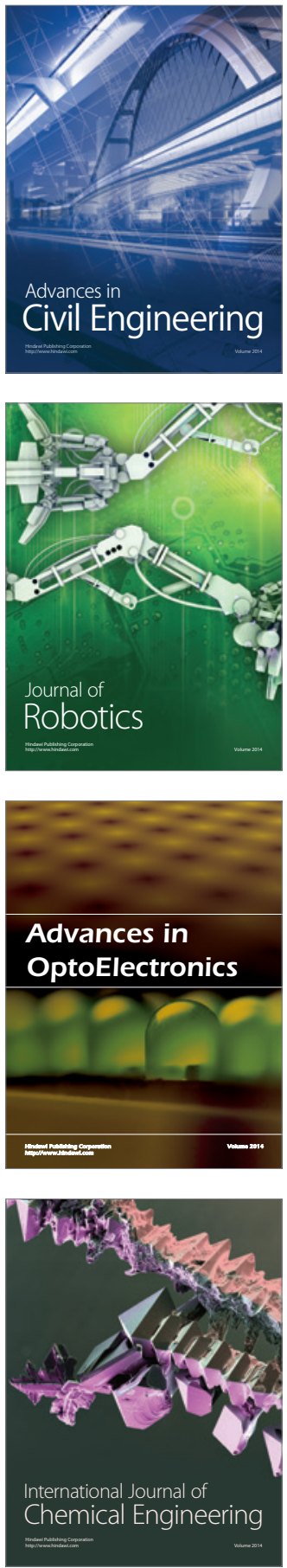

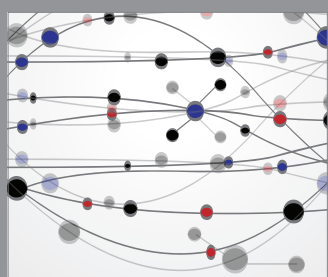

The Scientific World Journal

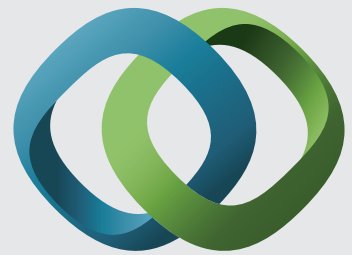

\section{Hindawi}

Submit your manuscripts at

https://www.hindawi.com
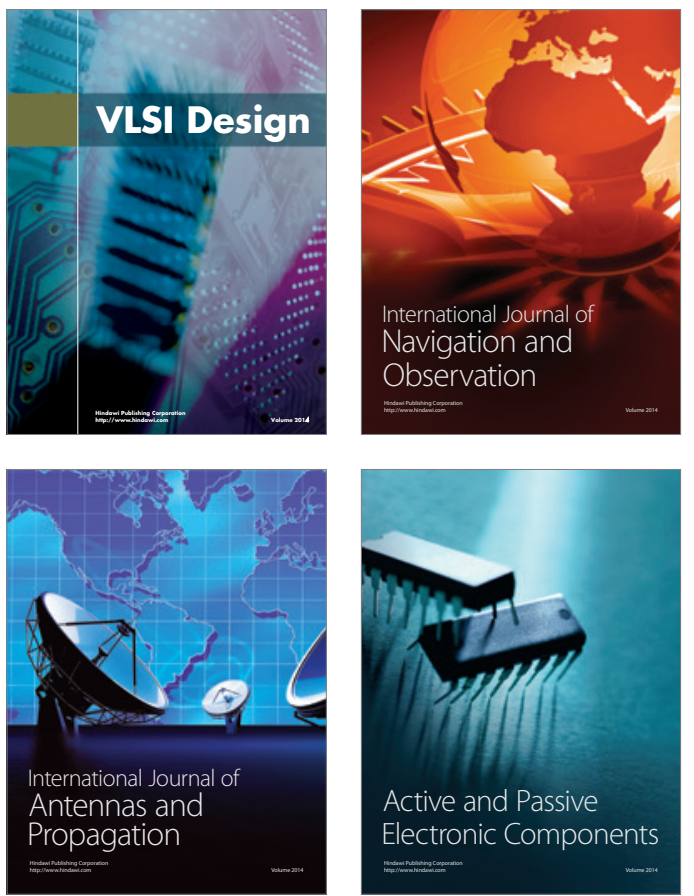
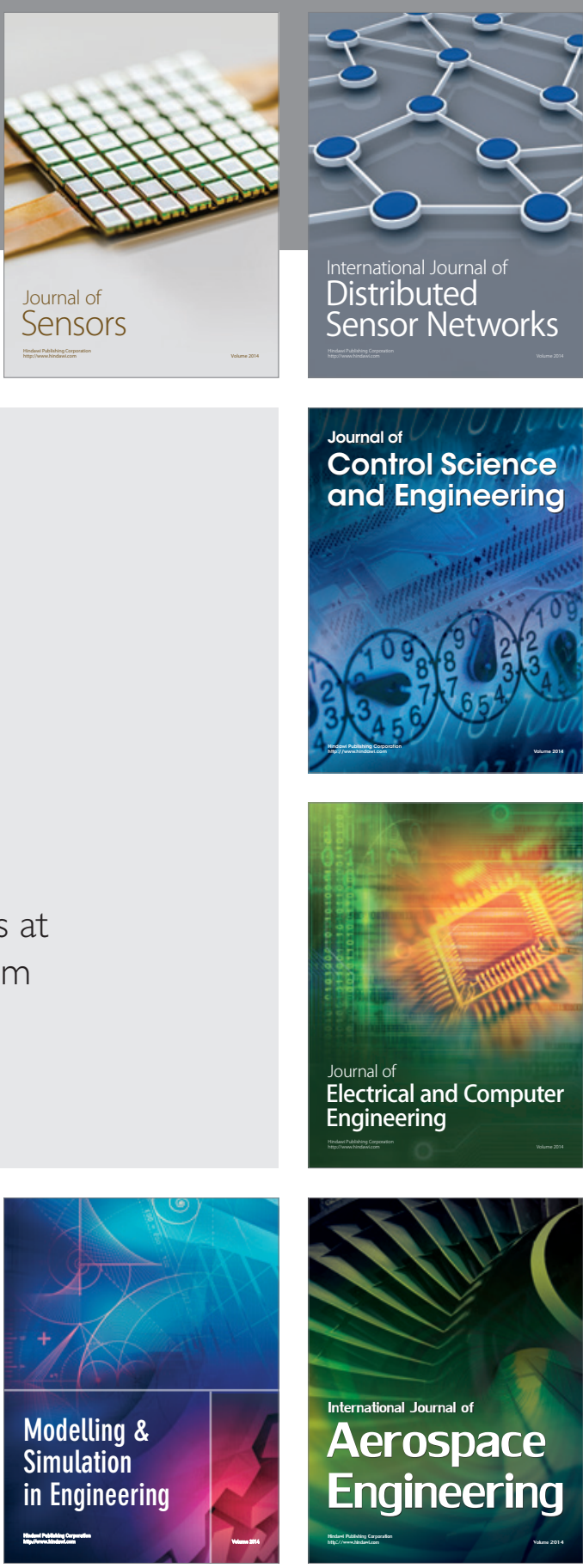

International Journal of

Distributed

Sensor Networks

$-$

Joumal of

Control Science

and Engineering
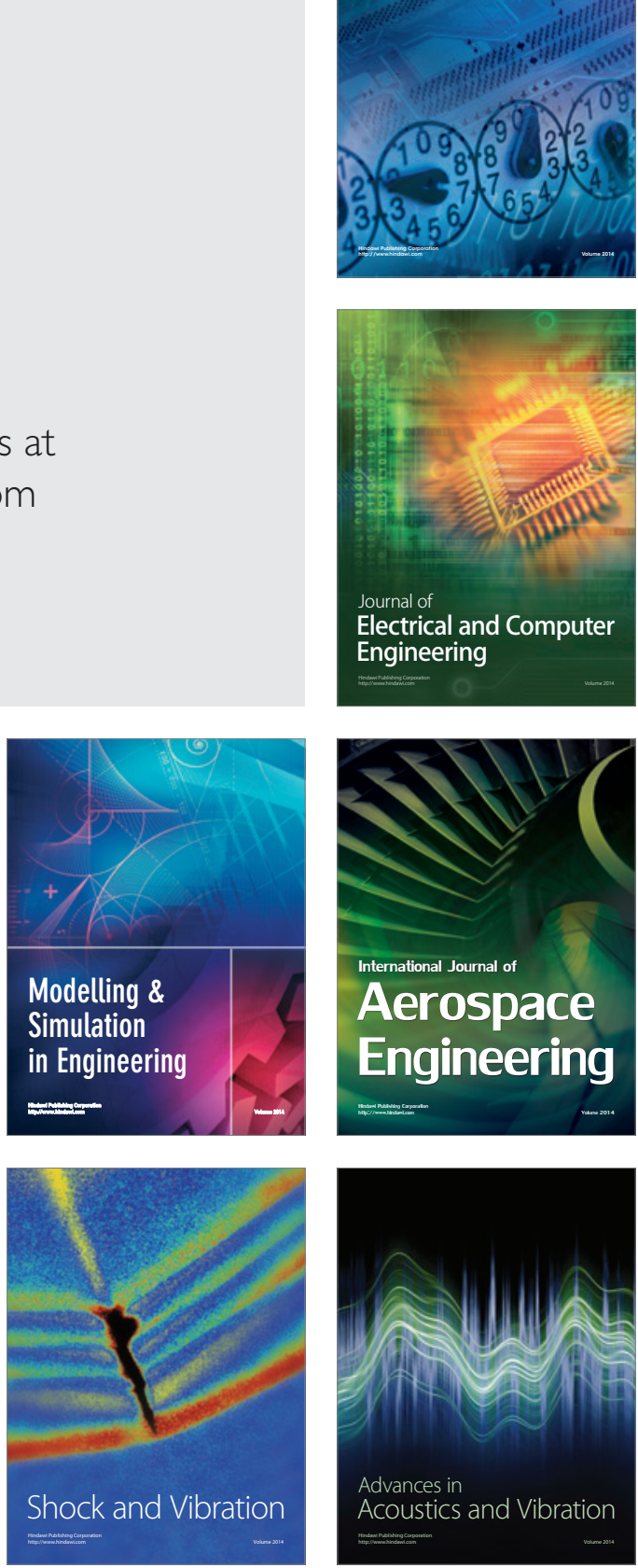